\title{
Investigation of Magneto-phonon Resonance in Graphene Monolayers
}

\author{
Le Thi Thu Phuong ${ }^{1}$, Tran Thi My Duyen ${ }^{1}$, Vo Thanh Lam $^{2}$, Bui Dinh Hoi ${ }^{1, *}$ \\ ${ }^{1}$ University of Education, Hue University, Hue City, Vietnam \\ ${ }^{2}$ Sai Gon University, Ho Chi Minh City, Vietnam
}

Received 11 October 2017

Revised 05 November 2017; Accepted 27 November 2017

\begin{abstract}
In this work, utilising the linear response theory we calculate the magneto conductivity (MC) in graphene monolayers, subjected to a static perpendicular magnetic field. The interaction of Dirac fermions with optical phonon via deformation potential is taken into account at high temperature. The dependence of the $\mathrm{MC}$ on the magnetic field shows resonant peaks that describe transitions of electrons between Landau levels via the resonant scattering with optical phonons. The effect of temperature on the MC is also obtained and discussed.
\end{abstract}

Keywords: Magnetophonon resonance, graphene, optical phonon.

\section{Introduction}

Magnetophonon resonance (MPR) arises from resonant phonon emission and absorption by electrons in semiconductors in high magnetic field [1-4]. The condition for the MPR has been obtained in bulk and conventional low-dimensional semiconductors as

$\omega_{o p}=M \omega_{c}$,

where $M=1,2,3, \ldots, \omega_{o p}$ and $\omega_{c}$ are, respectively, the optical phonon and cyclotron frequency. MPR provides detailed information on carrier effective mass and phonon frequency at higher temperatures, typically between liquid nitrogen and room temperature. Since the first discovery [5], graphene has attracted numerous interest because of its unique properties that make graphene a promising candidate for future electronics devices. Electrons in graphene can move with a very high speed which leads to relativistic description of their dynamics, their behavior is described by the twodimensional Dirac equation for massless fermions. The energy dispersion in graphene is linear near the Dirac points. In particular, electronic structure of graphene in magnetic field shows unusual behaviors. Unlike conventional low-dimensional semiconductors where electron Landau levels (LL) are proportional to magnetic field and equally spaced, the LLs in graphene are proportional to the

\footnotetext{
*Corresponding author. Tel.: 84-916666819.

Email: buidinhhoi@hueuni.edu.vn

https//doi.org/ 10.25073/2588-1124/vnumap.4235
} 
square root of magnetic field and their spacing depends on the indices of LLs. This unusual energy spectrum of electrons in graphene in magnetic field has been expected to result in many exceptional and fascinating physical properties, including magneto-transport properties. For example, the MPR condition in graphene may be fairly different from Eq. (1). In this work, utilising the linear response theory we calculate the magnetoconductivity (MC) in graphene monolayers subjected to a static perpendicular magnetic field. We only consider the scattering of electrons and optical phonons at $\mathrm{K}$ points and take account of arbitrary transitions between the energy levels. In the next section, we introduce basic formulae of calculation. Numerical results and discussion are presented in Sec. 3 . Finally, concluding remarks are given briefly in Sec. 4.

\section{Basic formulation}

For a many body system, let us consider the Hamiltonian [6]

$H=H_{0}+\lambda V-A . F(t)$,

where $H_{0}$ is the largest part of $H$ which can be diagonalized (analytically), $\lambda V$ is a binary-type interaction, assumed nondiagonal and small compared to $H_{0}$, and $-A . F(t)$ is the external field Hamiltonian with $A$ being an operator and $F(t)$ a generalized force. Based on this Hamiltonian, $\mathrm{K}$. Van Vliet and co-workers developed a general expression for the conductivity tensor in linear response theory using projection operator technique of Zwanzig [7] in which the conductivity was split into the diagonal and nondiagonal parts. The magneto-conductivity (MC) tensor can be calculated by relating it to the transition probability electron as

$$
\sigma_{\mu \mu}^{d}(0)=\frac{\beta e^{2}}{V_{0}} \sum_{\zeta, \zeta^{\prime}, s}\left\langle n_{\zeta}\right\rangle_{e q}\left(1-\left\langle n_{\zeta^{\prime}}\right\rangle_{e q}\right) W_{\zeta \zeta^{\prime}}\left(\chi_{\mu \zeta}-\chi_{\mu \zeta^{\prime}}\right)^{2},
$$

where $V_{0}$ is the normalization volume of the system, $\beta=1 / k_{B} T$ with $k_{B}$ being Boltzmann constant and $T$ the temperature, $W_{\zeta \zeta^{\prime}}$ is the binary transition rate, given by the Fermi "golden rule" and $\left\langle n_{\zeta}\right\rangle_{e q}$ is the Fermi-Dirac distribution function. For the electron-phonon interaction, the transition rate $W_{\zeta \zeta^{\prime}}$ takes the form

$$
W_{\zeta \zeta^{\prime}}=\sum_{\vec{q}}\left[Q\left(\zeta, \vec{q} \rightarrow \zeta^{\prime}\right)\left\langle N_{\vec{q}}\right\rangle_{e q}+Q\left(\zeta \rightarrow \zeta^{\prime}, \vec{q}\right)\left(1+\left\langle N_{\vec{q}}\right\rangle_{e q}\right)\right]
$$

where

$$
\begin{aligned}
& Q\left(\zeta, \vec{q} \rightarrow \zeta^{\prime}\right)=\frac{2 \pi}{\hbar}|C(\vec{q})|^{2}\left|\left\langle\zeta^{\prime}\left|e^{i \vec{q} \vec{r}}\right| \zeta\right\rangle\right|^{2} \delta\left(\varepsilon_{\zeta}-\varepsilon_{\zeta^{\prime}}+\hbar \omega_{\vec{q}}\right), \\
& Q\left(\zeta \rightarrow \zeta^{\prime}, \vec{q}\right)=\frac{2 \pi}{\hbar}|C(\vec{q})|^{2}\left|\left\langle\zeta^{\prime}\left|e^{-i \vec{q} \vec{r}}\right| \zeta\right\rangle\right|^{2} \delta\left(\varepsilon_{\zeta}-\varepsilon_{\zeta^{\prime}}-\hbar \omega_{\vec{q}}\right),
\end{aligned}
$$

with $Q\left(\zeta, \vec{q} \rightarrow \zeta^{\prime}\right), Q\left(\zeta \rightarrow \zeta^{\prime}, \vec{q}\right)$ correspond to absorption and emission of a phonon with wave vector $\vec{q}$, and energy $\hbar \omega_{\vec{q}}$, respectively, and $\left\langle N_{\vec{q}}\right\rangle_{e q}$ is the equilibrium distribution function of phonons.

We now apply the above expression of the MC to a graphene sheet placed in the $(x-y)$ plane, subjected to an uniform static magnetic field with strength $B$ oriented along the $z$-direction. The 
normalized wave function and the corresponding energy for a carrier (electron and hole) in the Landau gauge for the vector potential $A=(B x, 0)$ are written as [8]

$$
\begin{aligned}
& \psi_{n}(\vec{r})=\frac{C_{n}}{\sqrt{L}} e^{-i X y / l^{2}}\left[\begin{array}{c}
S_{n} \phi_{|n|-I}(x-X) \\
\phi_{|n|}(x-X)
\end{array}\right], \\
& \varepsilon_{n}=S_{n} \hbar \omega_{B} \sqrt{|n|},
\end{aligned}
$$

where

$$
\begin{aligned}
& C_{n}=\sqrt{\frac{\left(1+\delta_{n, 0}\right)}{2}}=\left\{\begin{array}{lll}
1 & \text { for } & (n=0) \\
\frac{1}{\sqrt{2}} & \text { for } & (n \neq 0),
\end{array}\right. \\
& S_{n}=\left\{\begin{array}{lll}
1 & \text { for } & (n>0) \\
0 & \text { for } & (n=0), \\
-1 & \text { for } & (n<0)
\end{array}\right. \\
& \phi_{|n|}(x)=\frac{i^{|n|}}{\sqrt{2^{|n|}|n| ! \sqrt{\pi} l}} \exp \left[-\frac{1}{2}\left(\frac{x}{l}\right)^{2}\right] H_{|n|}\left(\frac{x}{l}\right),
\end{aligned}
$$

with $n=0, \pm 1, \pm 2, \ldots$ being the Landau index, $H_{n}(x / l)$ is the $n$-th order Hermite polynomial, $x$ being the coordinate of the center of the carrier orbit, $\hbar \omega_{B}=\sqrt{2} \gamma / l$ is the effective magnetic energy with $\gamma=(\sqrt{3} / 2) a \gamma_{0}$ being the band parameter, and $a=0.246 \mathrm{~nm}$ being the lattice constant. The electronic states for a carrier are specified by the set of $\alpha=(n, X)$. To calculate the component $\sigma_{x x}$ of the $\mathrm{MC}$ from Eq. (3), we need following matrix elements [9]

$$
\begin{aligned}
& \chi_{\mu \zeta}=\langle\zeta|x| \zeta\rangle=l^{2} k_{y}, \\
& \chi_{\mu \zeta^{\prime}}=\left\langle\zeta^{\prime}|x| \zeta^{\prime}\right\rangle=l^{2} k_{y}^{\prime}, \\
& \left|\left\langle\zeta^{\prime}\left|e^{ \pm i \overrightarrow{q r}}\right| \zeta\right\rangle\right|^{2}=\left|J_{n n^{\prime}}(u)\right|^{2} \delta_{k_{y}, k_{y}^{\prime} \pm q_{y}} \\
& \left|J_{n n^{\prime}}(u)\right|^{2}=C_{n}^{2} C_{n^{\prime}}^{2} \frac{m !}{(m+j) !} e^{-u} u^{j}\left[L_{m}^{j}(u)+S_{n} S_{n^{\prime}} \sqrt{\frac{m+j}{m}} L_{m-l}^{j}(u)\right]^{2},
\end{aligned}
$$

where $L_{m}^{j}(u)$ is an associated Laguerre polynomial, $u=l^{2} q^{2} / 2, \quad q^{2}=q_{x}^{2}+q_{y}^{2}$, $m=\min \left(|n|,\left|n^{\prime}\right|\right), j=\| n|-| n^{\prime}||$. The MC tensor in graphene monolayers is written as [9]

$$
\sigma_{x x}=\frac{\beta e^{2}}{S_{0}} \sum_{\zeta, \zeta^{\prime}}\left\langle n_{\zeta}\right\rangle_{e q}\left(1-\left\langle n_{\zeta^{\prime}}\right\rangle_{e q}\right) W_{\zeta \zeta^{\prime}}\left(\chi_{x \zeta}-\chi_{x \zeta^{\prime}}\right)^{2}
$$

where $S_{0}$ is the normalization acreage of system, $W_{\zeta \zeta^{\prime}}$ is given by [10]

$$
W_{\zeta \zeta^{\prime}}=g_{s} g_{v} \sum_{\vec{q}} g\left(\theta_{\vec{k}, \vec{k}^{\prime}}\right)\left[Q\left(\zeta, \vec{q} \rightarrow \zeta^{\prime}\right)\left\langle N_{\vec{q}}\right\rangle_{e q}+Q\left(\zeta \rightarrow \zeta^{\prime}, \vec{q}\right)\left(1+\left\langle N_{\vec{q}}\right\rangle_{e q}\right)\right] \text {, }
$$


with $g_{v}=2$ and $g_{s}=2$ are the valley and spin degeneracy, respectively, $g(\theta)=(1+\cos \theta) / 2$ is the overlap integral of spinor wave functions, $Q\left(\zeta, \vec{q} \rightarrow \zeta^{\prime}\right)$ and $Q\left(\zeta \rightarrow \zeta^{\prime}, \vec{q}\right)$ are given by Eqs. (5) and (6).

Graphene has two atoms per unit cell, so it has four optical phonon modes. Because the contributions of $K$ - and $\Gamma$ - optical phonons are equivalent, so we consider only scattering of electrons with $K$-optical phonons. For the deformation potential scattering mechanism, we have

$$
|C(\vec{q})|^{2}=\frac{\hbar D_{o p}^{2}}{2 \rho L^{2} \omega_{K}}
$$

where $D_{o p}$ is the deformation potential constant, $\rho$ is the areal massdensity, $\omega_{K}$ is the $K$-optical phonon frequency.

Substituting Eq. (17) into Eq. (16) and using the notation $\left\langle n_{\zeta}\right\rangle_{e q} \equiv f_{n, k_{y}}$, we have

$$
\begin{aligned}
& \sigma_{x x}=\frac{2 \pi \beta e^{2} l^{4} D_{o p}^{2}}{\rho L^{2} \omega_{K} S_{0}} \sum_{n, k_{y}} \sum_{n^{\prime}, k_{y}^{\prime}} \sum_{\vec{q}}(1+\cos \theta) f_{n, k_{y}}\left(1-f_{n^{\prime}, k_{y}^{\prime}}\right)\left|J_{n n^{\prime}}(u)\right|^{2} \\
& \times\left\{N_{0} \delta_{k_{y}, k_{y}^{\prime}+q_{y}} \delta\left(\varepsilon_{n}-\varepsilon_{n^{\prime}}+\hbar \omega_{K}\right)\left(k_{y}-k_{y}^{\prime}\right)^{2}+\right. \\
& \left.+\left(1+N_{0}\right) \delta_{k_{y}, k_{y}^{\prime}-q_{y}} \delta\left(\varepsilon_{n}-\varepsilon_{n^{\prime}}-\hbar \omega_{K}\right)\left(k_{y}-k_{y}^{\prime}\right)^{2}\right\},
\end{aligned}
$$

Inserting Eq. (15) into Eq. (19) and peforming the integral over $\vec{q}$, we obtain the following expression of the transverse MC in graphene monolayers:

$$
\begin{aligned}
& \sigma_{x x}=\frac{\beta e^{2} D_{o p}^{2}}{2 \rho \omega_{K} l^{2}} \sum_{n, n^{\prime}} f_{n}\left(1-f_{n^{\prime}}\right) C_{n}^{2} C_{n^{\prime}}^{2} \\
& \times\left\{(2 m+j+l)-2 S_{n} S_{n^{\prime}} \sqrt{m(m+j)}+S_{n}^{2} S_{n^{\prime}}^{2}(2 m+j-l)\right\} \\
& \times\left\{N_{0} \delta\left(\varepsilon_{n}-\varepsilon_{n^{\prime}}+\hbar \omega_{K}\right)+\left(1+N_{o}\right) \delta\left(\varepsilon_{n}-\varepsilon_{n^{\prime}}-\hbar \omega_{K}\right)\right\} .
\end{aligned}
$$

The delta functions in Eq. (20) are divergent as their arguments equals to zero. To avoid this, we replace them phenomenology by Lorentzians as [10]

$$
\delta(\varepsilon)=\frac{\Gamma}{\pi\left(\varepsilon^{2}+\Gamma^{2}\right)},
$$

where $\Gamma_{K}=\hbar \omega_{B} \sqrt{W_{K}}$ is the level width, $W_{K}=\hbar^{2} D_{o p}^{2} / 8 \pi \rho \gamma^{2} \hbar \omega_{K}$ is the dimensionless parameter characterizing the scattering strength.

\section{Numerical results and discussion}

We have obtained analytic expression of the transverse $\mathrm{MC}$ in graphene monolayers when carriers are scattered by K-optical phonons. The above result will now be applied to numerically investigate physical behavoiurs of the transverse MC. The parameters used in computational calculations are as 
follows [10, 11]: $k_{B}=1.3807 \times 10^{-23} \mathrm{~J} / \mathrm{K}, a=0.246 \mathrm{~nm}, \quad \gamma_{0}=3.03 \mathrm{eV}, D_{o p}=1.4 \times 10^{9} \mathrm{eV} / \mathrm{cm}$, $\rho=7.7 \times 10^{-8} \mathrm{~g} / \mathrm{cm}^{2}, \hbar \omega_{0}=\hbar \omega_{K}=162 \mathrm{meV}, n=-4 \div 4, n^{\prime}=-4 \div 4$.

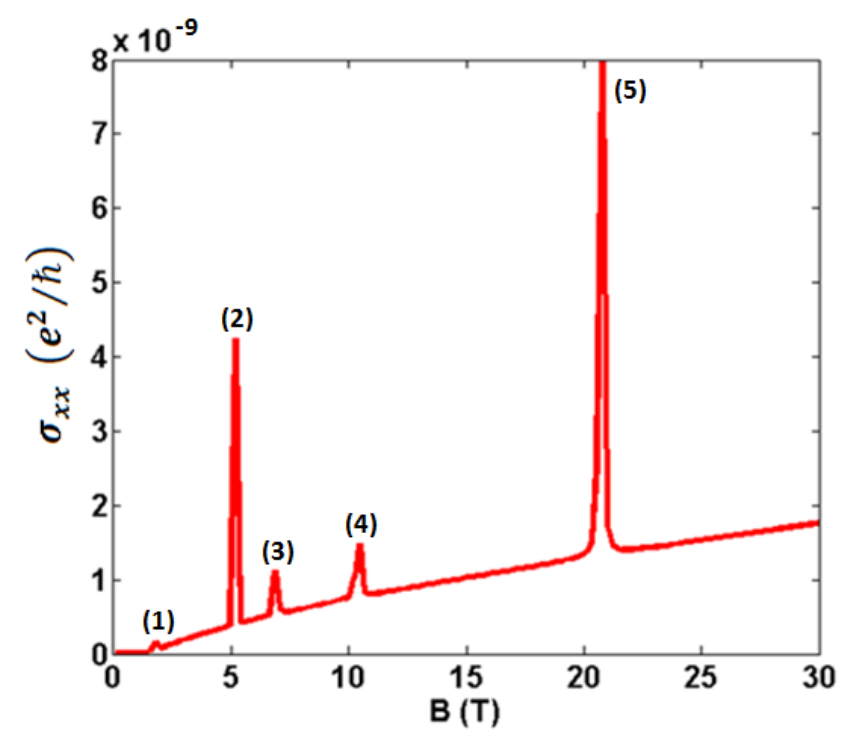

Figure 1. The dependence of the magnetoconductivity on the magnetic field. Here, $T=180 \mathrm{~K}$.

Figure 1 shows the dependence of the transverse $\mathrm{MC}$ on the magnetic field $B$ at $T=180 \mathrm{~K}$. It can be seen that there are 5 maximum peaks of the MC. By computational analysis, we can deduce their physical meanings as follows.

- Peak (1) appears at $B=1.789$ T satisfying the condition

$$
(1 \sqrt{|2|}-(-1) \sqrt{|-4|}) \hbar \omega_{B}-\hbar \omega_{K}=0 \text {, }
$$

it describes electron transition between LLs $n=2$ and $n^{\prime}=-4$ accompanied by emitting an optical phonon of energy $\hbar \omega_{K}$, or the condition

$$
(1 \sqrt{|4|}-(-1) \sqrt{|-2|}) \hbar \omega_{B}-\hbar \omega_{K}=0,
$$

describes electron transition between LLs $n=4$ and $n^{\prime}=-2$ accompanied by emitting an optical phonon of energy $\hbar \omega_{K}$, or the condition

$$
((-1) \sqrt{|-2|}-1 \sqrt{|4|}) \hbar \omega_{B}+\hbar \omega_{K}=0,
$$

describes electron transition between LLs $n=-2$ and $n^{\prime}=4$ accompanied by absorbing an optical phonon, or the condition

$$
((-1) \sqrt{|-4|}-1 \sqrt{|2|}) \hbar \omega_{B}+\hbar \omega_{K}=0,
$$

describes electron transition between LLs $n=-4$ and $n^{\prime}=2$ accompanied by absorbing an optical phonon. 

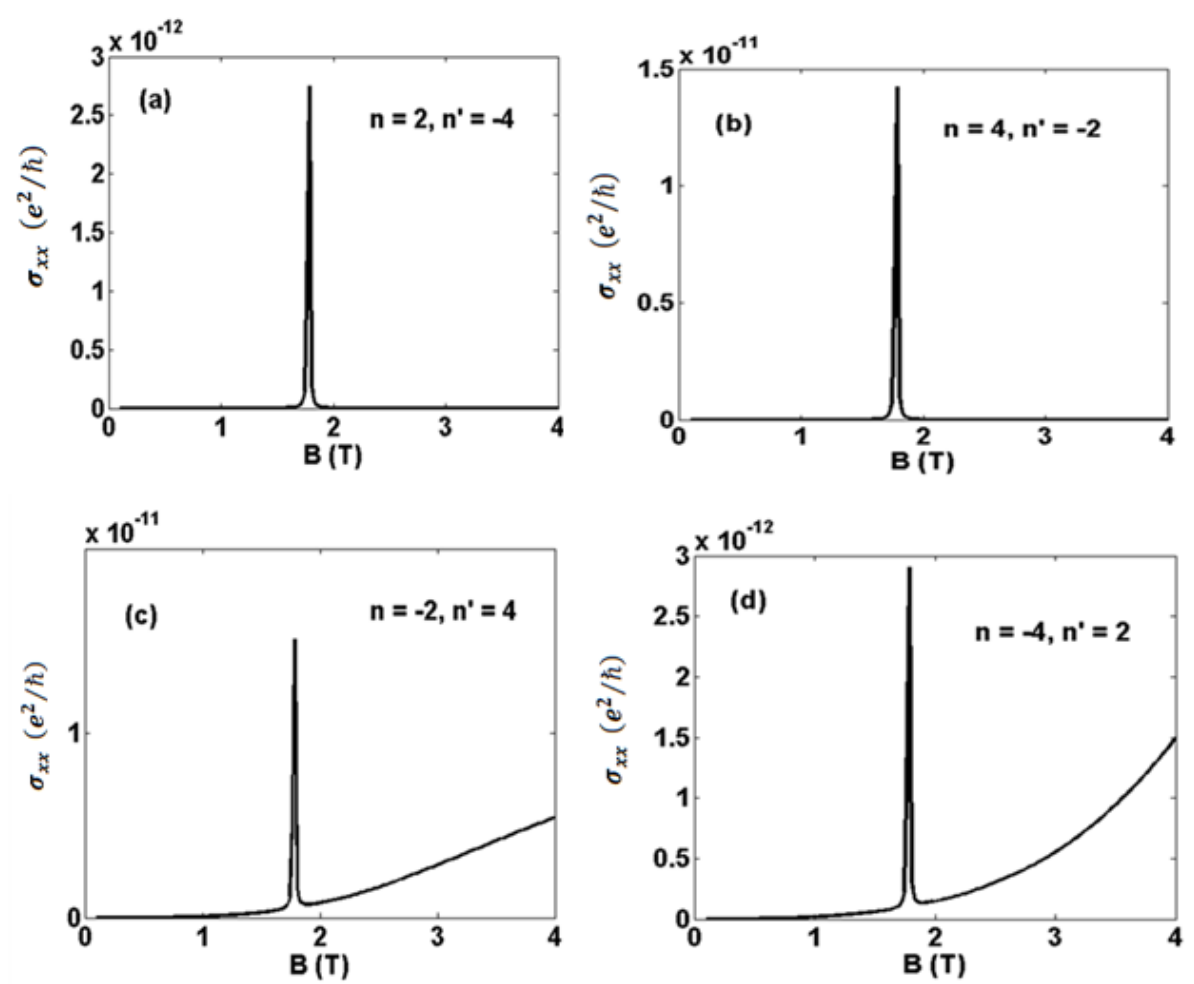

Figure 2. The MC as a function of the magnetic field for the transitions contributing to the resonance peak (1) in Figure 1. Figures a, b, c, d correspond to the possible transitions analyzed above, respectively.

Thus, peak (1) is contributed by four transitions of electrons in which two transitions with phonon absorption and two others with phonon emission, as shown in Figure. 2.

- Peak (2) located at $B=5.167 \mathrm{~T}$ is the contribution of the condition

$$
(1 \sqrt{|1|}-(-1) \sqrt{|-1|}) \hbar \omega_{B}-\hbar \omega_{K}=0 \text {, }
$$

describing electron transition between LLs $n=1$ and $n^{\prime}=-1$ accompanied by emitting an optical phonon of energy $\hbar \omega_{K}$, and the condition

$$
((-1) \sqrt{|-1|}-1 \sqrt{|1|}) \hbar \omega_{B}+\hbar \omega_{K}=0,
$$

describing electron transition between LLs $n=-1$ and $n^{\prime}=1$ accompanied by absorbing an optical phonon.

- Peak (3) appears at $B=6.856$ T satisfying the condition

$$
(1 \sqrt{|3|}-0 \sqrt{|0|}) \hbar \omega_{B}-\hbar \omega_{K}=0 \text {, }
$$

it describes electron transition between LLs $n=3$ and $n^{\prime}=0$ accompanied by emitting an optical phonon of energy $\hbar \omega_{K}$, or the condition

$$
(0 \sqrt{|0|}-(-1) \sqrt{|-3|}) \hbar \omega_{B}-\hbar \omega_{K}=0,
$$


describes electron transition between LLs $n=0$ and $n^{\prime}=-3$ accompanied by emitting an optical phonon of energy $\hbar \omega_{K}$, or the condition

$$
((-1) \sqrt{|-3|}-0 \sqrt{|O|}) \hbar \omega_{B}+\hbar \omega_{K}=0,
$$

describes electron transition between LLs $n=-3$ and $n^{\prime}=0$ accompanied by absorbing an optical phonon, or the condition

$$
(0 \sqrt{|0|}-1 \sqrt{|3|}) \hbar \omega_{B}+\hbar \omega_{K}=0,
$$

describes electron transition between LLs $n=0$ and $n^{\prime}=3$ accompanied by absorbing an optical phonon.

Thus, peak (3) arises from the contributions of the above four transitions of electrons in which two transitions with phonon absorption and two others with phonon emission.

- Peak (4) appears at $B=10.44 \mathrm{~T}$ satisfying the condition

$(1 \sqrt{|2|}-0 \sqrt{|O|}) \hbar \omega_{B}-\hbar \omega_{K}=0$,

it describes electron transition between LLs $n=2$ and $n^{\prime}=0$ accompanied by emitting an optical phonon of energy $\hbar \omega_{K}$, or the condition

$$
(0 \sqrt{|0|}-(-1) \sqrt{|-2|}) \hbar \omega_{B}-\hbar \omega_{K}=0,
$$

describes electron transition between LLs $n=0$ and $n^{\prime}=-2$ accompanied by emitting an optical phonon of energy $\hbar \omega_{K}$, or the condition

$$
((-1) \sqrt{|-2|}-0 \sqrt{|O|}) \hbar \omega_{B}+\hbar \omega_{K}=0
$$

describes electron transition between LLs $n=-2$ and $n^{\prime}=0$ accompanied by absorbing an optical phonon, or the condition

$$
(0 \sqrt{|0|}-1 \sqrt{|2|}) \hbar \omega_{B}+\hbar \omega_{K}=0,
$$
phonon.

describes electron transition between LLs $n=0$ and $n^{\prime}=2$ accompanied by absorbing an optical

Thus, peak (4) arises from the contributions of the above four transitions of electrons in which two transitions with phonon absorption and two others with phonon emission

- Peak (5) appears at $B=20.79$ T satisfying the condition

$$
(1 \sqrt{|1|}-0 \sqrt{|0|}) \hbar \omega_{B}-\hbar \omega_{K}=0 \text {, }
$$

it describes electron transitions between LLs $n=1$ and $n^{\prime}=0$ accompanied by emitting an optical phonon of energy $\hbar \omega_{K}$, or the condition

$$
(0 \sqrt{|0|}-(-1) \sqrt{|-1|}) \hbar \omega_{B}-\hbar \omega_{K}=0,
$$

describes electron transition between LLs $n=0$ and $n^{\prime}=-1$ accompanied by emitting an optical phonon of energy $\hbar \omega_{K}$, or the condition 


$$
((-1) \sqrt{|-1|}-0 \sqrt{|0|}) \hbar \omega_{B}+\hbar \omega_{K}=0,
$$

describes electron transition between LLs $n=-1$ and $n^{\prime}=0$ accompanied by absorbing an optical phonon, or the condition

$$
(0 \sqrt{|0|}-1 \sqrt{|1|}) \hbar \omega_{B}+\hbar \omega_{K}=0,
$$

describes electron transition between LLs $n=0$ and $n^{\prime}=1$ accompanied by absorbing an optical phonon.

Thus, peak (5) arises from the contributions of the above four transitions of electrons in which two transitions with phonon absorption and two others with phonon emission.

From the above results it can be deduced that the general condition for the maxima of the transverse $\mathrm{MC}$ is

$\varepsilon_{n}-\varepsilon_{n^{\prime}} \pm \hbar \omega_{K}=0$,

where $\varepsilon_{n}-\varepsilon_{n^{\prime}}=\left(S_{n} \sqrt{|n|}-S_{n^{\prime}} \sqrt{\left|n^{\prime}\right|}\right) \hbar \omega_{B},\left(+\hbar \omega_{K}\right)$ is for phonon absorption, $\left(-\hbar \omega_{K}\right)$ is for phonon emission. This condition is called the MPR condition in graphene monolayers. Also, it is possible to devide electron transitions into three types as follows:

The principal transitions are between $n=0$ and $n^{\prime}= \pm 1, \pm 2, \ldots$ (or $n^{\prime}=0$ and $n= \pm 1, \pm 2, \ldots$ ), in this case the condition (40) becomes, respectively

$$
-S_{n^{\prime}} \sqrt{\left|n^{\prime}\right|} \hbar \omega_{B} \pm \hbar \omega_{K}=0,
$$

or

$$
S_{n} \sqrt{|n|} \hbar \omega_{B} \pm \hbar \omega_{K}=0 .
$$

The symmetric transitions are between $n$ and $n^{\prime}=-n$, in this case the condition (40) becomes

$$
2 S_{n} \sqrt{|n|} \hbar \omega_{B} \pm \hbar \omega_{K}=0 .
$$

The asymmetric transitions are all other transitions, then the condition (40) becomes

$$
\mp\left(\sqrt{|n|}+\sqrt{\left|n^{\prime}\right|}\right) \hbar \omega_{B} \pm \hbar \omega_{K}=0 .
$$

The above conditions for MPR in graphene monolayers are consistent with the ones obtained previously by Mori N. and Ando T. [11] using Kubo formula in which the authors only considered the phonon absorption term in the conductivity. In this calculation, we consider both the phonon absorption and phonon emission.

In Figure 4 the $\mathrm{MC}$ is plotted versus temperature $T$ at different values of the magnetic field. We can see that the MC decreases as the temperature increases and reaches saturation when the temperature is very high. This can be explained by the increase of the probability of electron-phonon scattering with increasing the temperature, resulting in the decrease of the conductivity. This behavior is consistent with the temperature dependence of the conductivity in graphene obtained by S. V. Kryuchkov and co-workers in the work [12], in which the authors used the Boltzmann equation to calculate the MC and the Hall conductance for electron - optical phonon and electron - acoustic phonon interactions. 


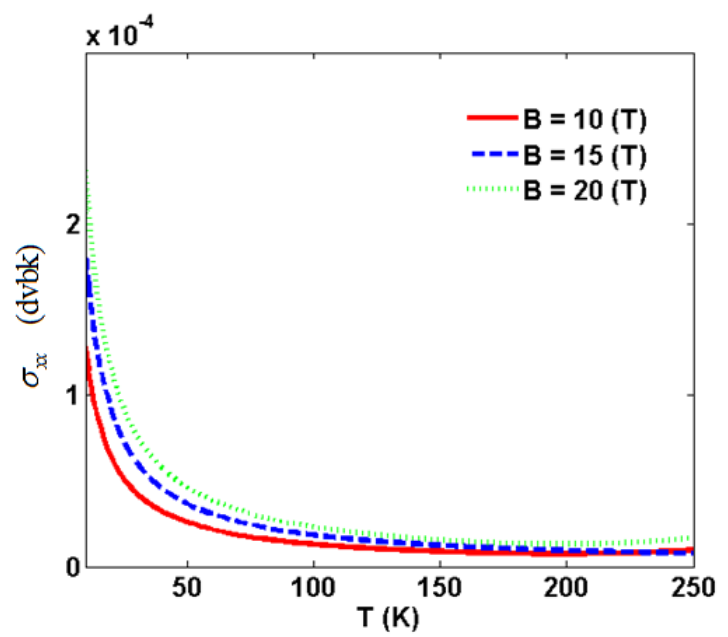

Figure 4. The magnetoconductivity verus temperature at different values of the magnetic field.

\section{Conclusions}

So far, we have calculated the transverse MC in monolayer graphene subjected to a perpendicupar magnetic field. The electron-optical phonon interaction is taken into account at high temperatures. The dependence of the transverse MC on the magnetic field shows MPR effect that arises from transitions of electrons between LLs via resonant scattering with optical phonons. The MPR conditions in the present calculation show the unsusual behaviour of Dirac fermions in graphene in comparison with the carriers in conventional semiconductors. Numerical results also show that the transverse MC decreases with increasing the temperature and reaches saturation at high temperature.

\section{Acknowledgements}

This research is funded by Vietnam National Foundation for Science and Technology Development (NAFOSTED) under Grant Number 103.01-2016.83.

\section{Appendix}

In this appendix, we present the detailed calculation to obtain Eq. (20) from Eq. (19). Let us rewrite again Eq. (19) as

$$
\begin{aligned}
& \sigma_{x x}=\frac{2 \pi \beta e^{2} l^{4} D_{o p}^{2}}{\rho L^{2} \omega_{0} S_{0}} \sum_{n, k_{y}} \sum_{n^{\prime}, k_{y}^{\prime}} \sum_{\vec{q}}(1+\cos \theta) f_{n, k_{y}}\left(1-f_{n^{\prime}, k_{y}^{\prime}}\right)\left|J_{n n^{\prime}}(u)\right|^{2} \\
& \times\left\{N_{0} \delta_{k_{y}, k_{y}^{\prime}+q_{y}} \delta\left(\varepsilon_{n}-\varepsilon_{n^{\prime}}+\hbar \omega_{K}\right)\left(k_{y}-k_{y}^{\prime}\right)^{2}+\right. \\
& \left.+\left(1+N_{0}\right) \delta_{k_{y}, k_{y}^{\prime}-q_{y}} \delta\left(\varepsilon_{n}-\varepsilon_{n^{\prime}}-\hbar \omega_{K}\right)\left(k_{y}-k_{y}^{\prime}\right)^{2}\right\} .
\end{aligned}
$$

Firstly, taking the summation over $k_{y}^{\prime}$, we have 


$$
\begin{aligned}
& \sigma_{x x}=\frac{2 \pi \beta e^{2} l^{4} D_{o p}^{2}}{\rho L^{2} \omega_{0} S_{0}} \sum_{n, k_{y}} \sum_{n^{\prime}} \sum_{\vec{q}}(1+\cos \theta) f_{n}\left(1-f_{n^{\prime}}\right)\left|J_{n n^{\prime}}(u)\right|^{2} \\
& \left\{N_{o} \delta\left(\varepsilon_{n}-\varepsilon_{n^{\prime}}+\hbar \omega_{K}\right)+\left(1+N_{o}\right) \delta\left(\varepsilon_{n}-\varepsilon_{n^{\prime}}-\hbar \omega_{K}\right)\right\} q_{y}^{2} .
\end{aligned}
$$

Because the $x$ and $y$ directions are symmetric, so we also have

$$
\begin{aligned}
& \sigma_{y y}=\frac{2 \pi \beta e^{2} l^{4} D_{o p}^{2}}{\rho L^{2} \omega_{0} S_{0}} \sum_{n, k_{x}} \sum_{n^{\prime}} \sum_{\vec{q}}(1+\cos \theta) f_{n}\left(1-f_{n^{\prime}}\right)\left|J_{n n^{\prime}}(u)\right|^{2} \\
& \left\{N_{o} \delta\left(\varepsilon_{n}-\varepsilon_{n^{\prime}}+\hbar \omega_{K}\right)+\left(1+N_{0}\right) \delta\left(\varepsilon_{n}-\varepsilon_{n^{\prime}}-\hbar \omega_{K}\right)\right\} q_{x}^{2} .
\end{aligned}
$$

Then, we can write

$$
\sigma_{x x}=\frac{\sigma_{x x}+\sigma_{y y}}{2} \text {. }
$$

Inserting (A.2) and (A.3) into (A.4), we have

$$
\begin{aligned}
& \sigma_{x x}=\frac{\pi \beta e^{2} l^{4} D_{o p}^{2}}{\rho L^{2} \omega_{0} S_{0}} \sum_{n, k_{y}} \sum_{n^{\prime}} \sum_{\vec{q}}(1+\cos \theta) f_{n}\left(1-f_{n^{\prime}}\right)\left|J_{n n^{\prime}}(u)\right|^{2} \\
& \left\{N_{0} \delta\left(\varepsilon_{n}-\varepsilon_{n^{\prime}}+\hbar \omega_{K}\right)+\left(1+N_{0}\right) \delta\left(\varepsilon_{n}-\varepsilon_{n^{\prime}}-\hbar \omega_{K}\right)\right\}\left(q_{x}^{2}+q_{y}^{2}\right) \\
& =\frac{\pi \beta e^{2} l^{4} D_{o p}^{2}}{\rho L^{2} \omega_{0} S_{0}} \sum_{n, k_{y}} \sum_{n^{\prime}} \sum_{\vec{q}}(1+\cos \theta) f_{n}\left(1-f_{n^{\prime}}\right)\left|J_{n n^{\prime}}(u)\right|^{2} \\
& \left\{N_{0} \delta\left(\varepsilon_{n}-\varepsilon_{n^{\prime}}+\hbar \omega_{K}\right)+\left(1+N_{0}\right) \delta\left(\varepsilon_{n}-\varepsilon_{n^{\prime}}-\hbar \omega_{K}\right)\right\} q^{2} .
\end{aligned}
$$

Transforming the summations over $q$ and $k_{y}$ to integrals as follows

$\sum_{\vec{q}} \rightarrow \frac{S_{0}}{(2 \pi)^{2}} \int_{0}^{\infty} q d q \int_{0}^{2 \pi} d \varphi=\frac{S_{0}}{2 \pi} \int_{0}^{\infty} q d q$,

$$
\sum_{k_{y}} \rightarrow \frac{L_{y}}{2 \pi} \int_{-L_{x} / 2 l^{2}}^{L_{x} / 2 l^{2}} d k_{y} \int_{0}^{\pi} d \theta=\frac{L_{x} L_{y}}{2 \pi l^{2}} \int_{0}^{\pi} d \theta,
$$

the expression (A.5) becomes

$$
\begin{aligned}
& \sigma_{x x}=\frac{\beta e^{2} l^{2} D_{o p}^{2}}{4 \pi \rho \omega_{0}} \sum_{n, n^{\prime}} f_{n}\left(1-f_{n^{\prime}}\right) \int_{0}^{\pi}(1+\cos \theta) d \theta \int_{0}^{\infty} q^{3}\left|J_{n n^{\prime}}(u)\right|^{2} d q \\
& \times\left\{N_{0} \delta\left(\varepsilon_{n}-\varepsilon_{n^{\prime}}+\hbar \omega_{K}\right)+\left(1+N_{0}\right) \delta\left(\varepsilon_{n}-\varepsilon_{n^{\prime}}-\hbar \omega_{K}\right)\right\} .
\end{aligned}
$$

We will calculate integrals in (A.8) as follows

$$
A=\int_{0}^{\pi}(1+\cos \theta) d \theta=\pi
$$

For the integral over $q$

$$
B=\int_{0}^{\infty} q^{3}\left|J_{n n^{\prime}}(u)\right|^{2} d q
$$

setting 
$u=\frac{l^{2} q^{2}}{2} \Rightarrow q^{2}=\frac{2 u}{l^{2}} \Rightarrow q d q=\frac{d u}{l^{2}}$,

we have

$$
\begin{aligned}
& B=\int_{0}^{\infty} \frac{2 u}{l^{2}} \frac{d u}{l^{2}} C_{n}^{2} C_{n^{\prime}}^{2} \frac{m !}{(m+j) !} e^{-u} u^{j}\left[L_{m}^{j}(u)+S_{n} S_{n^{\prime}} \sqrt{\frac{m+j}{m}} L_{m-l}^{j}(u)\right]^{2} \\
& =\frac{2 C_{n}^{2} C_{n^{\prime}}^{2}}{l^{4}} \int_{0}^{\infty} \frac{m !}{(m+j) !} e^{-u} u^{j+1}\left\{\left[L_{m}^{j}(u)\right]^{2}+2 L_{m}^{j}(u) S_{n} S_{n^{\prime}} \sqrt{\frac{m+j}{m}} L_{m-1}^{j}(u)+\frac{m+j}{m} S_{n}^{2} S_{n^{\prime}}^{2}\left[L_{m-1}^{j}(u)\right]^{2}\right\} d u \\
& =\frac{2 C_{n}^{2} C_{n^{\prime}}^{2}}{l^{4}}\left\{\frac{m !}{(m+j) !} \int_{0}^{\infty} e^{-u} u^{j+1}\left[L_{m}^{j}(u)\right]^{2} d u\right\} \\
& +\frac{2 C_{n}^{2} C_{n^{\prime}}^{2}}{l^{4}} 2 S_{n} S_{n^{\prime}}\left\{\frac{m !}{(m+j) !} \sqrt{\frac{m+j}{m}} \int_{0}^{\infty} e^{-u} u^{j+l} L_{m}^{j}(u) L_{m-l}^{j}(u) d u\right\} \\
& +\frac{2 C_{n}^{2} C_{n^{\prime}}^{2}}{l^{4}} S_{n}^{2} S_{n^{\prime}}^{2}\left\{\frac{(m-1) !}{(m+j-l) !} \int_{0}^{\infty} e^{-u} u^{j+1}\left[L_{m-l}^{j}(u)\right]^{2} d u\right\} .
\end{aligned}
$$

Using following formulae [13]

$$
\begin{aligned}
& B_{1}=\frac{m !}{(m+j) !} \int_{0}^{\infty} e^{-u} u^{j+1}\left[L_{m}^{j}(u)\right]^{2} d u=2 m+j+1, \\
& B_{2}=\frac{m !}{(m+j) !} \sqrt{\frac{m+j}{m}} \int_{0}^{\infty} e^{-u} u^{j+1} L_{m}^{j}(u) L_{m-l}^{j}(u) d u=-\sqrt{m(m+j)}, \\
& B_{3}=\frac{(m-1) !}{(m+j-1) !} \int_{0}^{\infty} e^{-u} u^{j+1}\left[L_{m-l}^{j}(u)\right]^{2} d u=2(m-1)+j+1=2 m+j-1,
\end{aligned}
$$

we arrive at

$$
B=\frac{2}{l^{4}} C_{n}^{2} C_{n^{\prime}}^{2}\left\{(2 m+j+1)-2 S_{n} S_{n^{\prime}} \sqrt{m(m+j)}+S_{n}^{2} S_{n^{\prime}}^{2}(2 m+j-1)\right\} .
$$

Finally, inserting (A.9) and (A.12) into (A.8) we obtain the explicit expression for the magnetoconductivity as

$$
\begin{aligned}
& \sigma_{x x}=\frac{\beta e^{2} D_{o p}^{2}}{2 \rho \omega_{0} l^{2}} \sum_{n, n^{\prime}} f_{n}\left(1-f_{n^{\prime}}\right) C_{n}^{2} C_{n^{\prime}}^{2} \\
& \times\left\{(2 m+j+1)-2 S_{n} S_{n^{\prime}} \sqrt{m(m+j)}+S_{n}^{2} S_{n^{\prime}}^{2}(2 m+j-1)\right\} \\
& \times\left\{N_{0} \delta\left(\varepsilon_{n}-\varepsilon_{n^{\prime}}+\hbar \omega_{k}\right)+\left(1+N_{0}\right) \delta\left(\varepsilon_{n}-\varepsilon_{n^{\prime}}-\hbar \omega_{k}\right)\right\} .
\end{aligned}
$$

\section{References}

[1] R. A. Stradling and R. A. Wood, The magnetophonon effect in III-V semiconducting compounds, J. Phys. C 1 (1968) 1711. 
[2] R. L. Peterson: in Semiconductors and Semimetals, ed. R. K. Willardson and A. C. Beer, Academic, New York, 1975, Vol. 10, p. 221

[3] R. J. Nicholas, The magnetophonon effect, Prog. Quantum Electron. 10 (1985) 1.

[4] C. Hamaguchi and N. Mori, Magnetophonon resonance in semiconductors, Physica B 164 (1990).

[5] K. S. Novoselov, A. K. Geim, S. V. Morozov, D. Jiang, Y. Zhang, S. V. Dubonos, I. V. Grigorieva, and A. A. Firsov, Electric Field Effect in Atomically Thin Carbon Films, Science 306, 666 (2004).

[6] P. Vasilopoulos, M. Charbonneau, and C. M. Van Vliet, Linear and nonlinear electrical conduction in quasi-twodimensional quantum wells, Phys. Rev. B 35(3) (1987) 1334.

[7] M. Charbonneau, K. M. Van Vliet, and P. Vasilopoulos, Linear response theory revisited III : One-body reponse formulas and generalized Boltzmann equations, J. Math. Phys. 23 (1982) 318.

[8] T. Ando, Magnetic Oscillation of Optical Phonon in Graphene, J. Phys. Soc. Jpn. 76(2), 024712 (2007).

[9] M. Ning, Z. Shengli, and L. Daqing, Mechanical control over valley magnetotransport in strained graphene, Physics Letters A 380 (2016) 1884.

[10] H. V. Phuc and N. N. Hieu, Nonlinear optical absorption in graphene via two-photon absorption process, Opt. Commun. 344 (2015) 12.

[11] N. Mori and T. Ando, Magnetophonon Resonance in Monolayer Graphene, J. Phys. Soc. Jpn. 80 (2011) 044706.

[12] S. V. Kryuchkov, E. I. Kukhar', and D. V. Zav'yalov, Conductivity of the graphene in the transversal magnetic field: Relaxation time approximation with Monte-Carlo method, Physica E 53 (2013) 124.

[13] I. S. Gradshteyn and I. M. Ryzhik, Table of Integrals, Series, and Products, Academic Press, seventh English edition, 2007. 